\title{
Growth, Structural, Optical, Mechanical and Dielectric Characterization of Diammonium Hydrogen Phosphate (DAHP) Single Crystals
}

\author{
P. Selvarajan ${ }^{a^{*}}$, N. Joseph John ${ }^{\mathrm{b}}$ and C.K. Mahadevan ${ }^{\mathrm{c}}$ \\ ${ }^{a}$ Department of Physics, Aditanar College of Arts and Science, Tiruchendur-628216, India. \\ ${ }^{\mathrm{b}}$ Department of Physics, Malankara Catholic College, Mariagiri, Tamil Nadu- 629153, India \\ ${ }^{c}$ Physcis Research Centre, S.T. Hindu College, Nagercoil-629003, Tamil Nadu, India. \\ *Corresponding Author: pselvarajanphy@yahoo.co.in
}

\begin{abstract}
Solubility studies of Diammonium Hydrogen Phosphate(DAHP) in de-ionized water were performed at various temperatures in the range $30-50{ }^{\circ} \mathrm{C}$. Single crystals of DAHP were grown from aqueous solutions by slow evaporation technique. Structural characterization of the grown crystals has been carried out by powder and single crystal X-ray diffraction (XRD) methods. DAHP crystals crystallize in monoclinic structure with the space group $P 2_{1} / c$. Second harmonic generation (SHG) for the material of this work was confirmed using Nd: YAG laser. The UV-Visible spectrum show that the grown crystals have wide optical transparency in the entire visible region. The dielectric measurements were made for grown DAHP crystal at the frequencies of $100 \mathrm{~Hz}$ and $1 \mathrm{KHz}$ and in the temperature range 30-150 ${ }^{\circ} \mathrm{C}$. Vickers microhardness value of DAHP crystal is found to increase with increase in load. Value of density of the grown DAHP crystal has been measured and it is well matched with the data of XRD studies.
\end{abstract}

MCCCLXXIX

Keywords: Optical Materials; Solubility; Crystal Growth; XRD; hardness; dielectric constant; SHG;

\section{INTRODUCTION}

The search for new conversion materials for various device applications has led to discovery of many organic, inorganic and semi-organic Nonlinear Optical (NLO) materials, which have potential applications in optoelectronics, Second Harmonic Generation (SHG), optical storage, optical communication, photonics, electro-optic modulation, optical parametric amplifiers, optical image processing, etc [1-4]. The salts of phosphate group such as 
Disodium Hydrogen Phosphate (DSHP), Trisodium Hydrogen Phosphate (TSHP) and Potassium Dihydrogen Phosphate (KDP) are reported to be NLO materials [5, 6]. Diammonium Hydrogen Phosphate (DAHP ) crystal belongs to the phosphate group and the hydrated nature of this crystal creates hydrogen bonding and this leads to have good optical properties. Solid DAHP loses ammonia readily on heating, for this reason it has been little studied at elevated temperatures. Many ammonium salts have characteristic transition temperatures for a wide temperature range [7]. X-ray studies of the title compound were reported by Smith et al and Khan et al $[8,9]$. Watton et al had carried out NMR and thermal studies of DAHP sample and reported that the material undergoes characteristic phase transitions, resulting in dramatic changes in many physical properties as temperature is changed [10]. Rotational Echo Double Resonance (REDOR) measurements on polycrystalline sample of diammonium hydrogen phosphate were reported by Gil Goobes et al [11]. Since few properties of DAHP crystals have been reported in the literature, it is planned to carry out a systematic study on various properties of the material in this work. Hence, the aim of this paper is to report the results of growth, solubility studies, structural, microhardness, UV-Visible-NIR transmittance studies of DAHP crystals. Also measurements of density, melting point, dielectric constant, dielectric loss of the grown crystals of DAHP are reported and discussed.

\section{EXPERIMENTAL PROCEDURE}

\subsection{Experimental for Solubility and Growth}

The commercially available Analar Reagent (AR) grade sample of Diammonium Hydrogen Phosphate (DAHP) was purchased from Merck India and it was further purified by repeated re-crystallization process for three times using de-ionzed water as the solvent. In order to obtain crystals of high quality, purification of starting material was found to be an important step and hence the re-crystallized salt was used for the growth of crystals of DAHP. Before the growth of single crystals of DAHP, solubility study was carried out using a hotplate magnetic stirrer and a digital thermometer. A voltage regulator was attached with hotplate magnetic stirrer in order to maintain the temperature constant (here accuracy is $\pm 0.1{ }^{\circ} \mathrm{C}$ ). Initially, the temperature was maintained at $30{ }^{\circ} \mathrm{C}$. The re-crystallized salt of DAHP was added step by step to $50 \mathrm{ml}$ of de-ionized water in an air-tight container kept on the hot-plate magnetic stirrer and stirring was continued till a small precipitate was formed. This gave confirmation of supersaturated condition of the solution. Then, $25 \mathrm{ml}$ of the solution was pipetted out and taken in a petri dish and it was warmed up till the solvent was evaporated out. By measuring the amount of salt present in the petri dish, the solubility ( in $\mathrm{g} / 100 \mathrm{ml}$ ) of DAHP in de-ionized water was determined gravimetrically [12]. The same procedure was followed to find solubility of DAHP sample at other temperatures such as $35,40,45$ and $50{ }^{\circ} \mathrm{C}$.

Single crystals of DAHP were grown by solution method with slow solvent evaporation technique at room temperature $\left(30^{\circ} \mathrm{C}\right)$. In accordance with the solubility data, the saturated solution of the re-crystallized salt of DAHP was prepared and it was constantly stirred for 
about 2 hours using a magnetic stirrer and were filtered using 4 micro Whatmann filter papers. Then the filtered solution was kept in a borosil beaker covered with a porous paper and kept in a dust-free atmosphere. The crystals were harvested after a period of about 30 days.

\subsection{Characterization techniques}

Single crystal XRD data for the grown single crystals of DAHP were collected using an ENRAF NONIUS CAD4 diffractometer with $\mathrm{MoK}_{\alpha}$ radiation $(\lambda=0.71073 \AA$ ). Powder X-ray diffraction pattern of the sample was obtained using a powder X-ray diffractometer (PANalytical Model, Nickel filtered $\mathrm{Cu} \mathrm{K}$ radiations $(\lambda=1.54056 \AA$ ) at $35 \mathrm{KV}, 10 \mathrm{~mA}$ ). The sample was scanned over the required range for $2 \Theta$ values $\left(10-70^{\circ}\right)$. The crystalline phase of the sample was identified from the crystallographic parameters such as $2 \theta$, relative intensity and hkl values. A Varian Cary 5E UV-Visible-NIR spectrophotometer was used for optical transmission studies in the range 190-1100 nm. The thermogravimetric and differential thermal analyses (TG/DTA) of the sample were carried out simultaneously using a Perkin Elmer thermal analyzer in nitrogen atmosphere at a heating rate of $10^{\circ} \mathrm{C} /$ minute for a temperature range of $35-1000^{\circ} \mathrm{C}$.

Second Harmonic Generation (SHG) test for the grown sample was performed by the powder technique of Kurtz and Perry [13] using a pulsed Nd:YAG laser (Model: YG501C, $\lambda=1064 \mathrm{~nm}$ ). Pulse energy of $4 \mathrm{~mJ} /$ pulse, pulse width of $10 \mathrm{~ns}$ and repetition rate of $10 \mathrm{~Hz}$ were used. The grown crystals were ground to powder of grain size 1500-1800 $\mu \mathrm{m}$ and the input laser beam was passed through IR reflector and directed on the powdered sample packed in a capillary tube. Mirocrystalline material of KDP was used as reference in this experiment. SHG from the sample was detected using an optical cable attached to a fluorescence spectroscope (Model: DID A-512 G/R).

Density of the grown crystal was determined by floatation method. Carbon tetrachloride of density $1.594 \mathrm{~g} / \mathrm{cc}$ and bromoform of density $2.890 \mathrm{~g} / \mathrm{cc}$ were used for this purpose. $10 \mathrm{ml}$ of carbon tetrachloride was taken in a specific gravity bottle and a small piece of the grown crystal was dropped into it. The crystal was at rest in the bottom of the bottle, then bromoform was gradually added until the crystal was in the suspended state. Now the density of crystal is equal to the density of solution. The density was calculated using the relation $\rho=\left(w_{3}-w_{1}\right) /\left(w_{2}-w_{1}\right) \quad$ where $w_{1}$ is the weight of empty specific gravity bottle, $w_{2}$ is the weight of the specific gravity bottle with full of water and $w_{3}$ is the weight of the specific gravity bottle with full of the mixture of bromoform and carbon tetrachloride [14].

The melting point of the grown crystal was measured using a melting point apparatus (Model: Tempo 120). Microhardness study of the grown crystals was carried out using Leitz Weitzler hardness tester fitted with a diamond indenter. Smooth, flat surface was selected and subjected to this study on the (100) plane of DAHP crystal. Indentations were made for various loads from $20 \mathrm{~g}$ to $160 \mathrm{~g}$. Several trials of indentation were carried out and the 
average diagonal lengths were measured for an indentation time of 10 seconds. The Vickers micro hardness number was calculated using the relation $\mathrm{H}_{\mathrm{v}}=1.8544 \mathrm{P} / \mathrm{d} \quad \mathrm{kg} / \mathrm{mm}^{2}$ where $\mathrm{P}$ is the applied load and $\mathrm{d}$ is the diagonal length of the indentation impression [15].

A sample crystal having a thickness about $4 \mathrm{~mm}$ was polished for dielectric measurements. Opposite faces of the sample crystal was coated with good quality silver paste to obtain a good ohmic contact with the electrodes. The capacitance and dielectric loss factor $(\tan \delta)$ were measured using the conventional two probe technique at frequencies of $100 \mathrm{~Hz}$ and 1 $\mathrm{KHz}$ and in the temperature range $30-150{ }^{\circ} \mathrm{C}$ using an LCR meter (Model APLAB) The dielectric constant of the crystal was calculated using the relation (as the crystal area was smaller than the plate area of the cell).

$$
\varepsilon_{\mathrm{r}}=\left\{\frac{\mathrm{C}_{\text {crys }}-\mathrm{C}_{\text {air }}\left(1-\mathrm{A}_{\text {crys }} / \mathrm{A}_{\text {air }}\right)}{\mathrm{C}_{\text {air }}}\right\} \frac{\left(\mathrm{A}_{\text {air }}\right)}{\left(\mathrm{A}_{\text {crys }}\right)}
$$

where $\mathrm{C}_{\text {crys }}$ is the capacitance with crystal (including air), $\mathrm{C}_{\text {air }}$ is the capacitance of air, $\mathrm{A}_{\text {crys }}$ is the area of the crystal touching the electrode and $A_{\text {air }}$ is the area of the electrode[16, 17]

\section{RESULTS AND DISCUSSION}

\subsection{Solubility and Growth}

Finding solubility of a material in a solvent is a useful analysis to grow bulk single crystals from solution. The size of a crystal depends on the amount of material available in the solution which in turn is decided by the solubility of the material in that solvent. To grow bulk crystals from solution growth technique, selection of solvent is an important step. To select a suitable solvent, the solubility of DAHP in different solvents such as water, ethanol and methanol were determined. Solubility is very low in ethanol and methanol and it is high in de-ionized water. Hence de-ionized water was used as solvent in this work. Solubility curve for DAHP sample at various temperatures ranging from 30 to $50{ }^{\circ} \mathrm{C}$ is shown in Fig. 1. Here solubility is defined as the amount of solute in grams present in $100 \mathrm{ml}$ of saturated solution at a particular temperature and it corresponds to saturation between a solid and its solution at given temperature and pressure. It is observed from the results that the solubility increases with temperature for the sample. Since solubility increases with temperature, the sample has positive temperature coefficient of solubility. Hence DAHP crystals can be grown by slow evaporation method. The harvested crystals of DAHP are displayed in Fig. 2. The grown crystals are found stable, non-hygroscopic at ambient temperature, colourless, transparent and have well defined appearance. Since the temperature was not completely kept constant during the growth of the crystals in the present work, there are slight morphological changes among the grown crystals. The dimensions of the biggest DAHP crystal harvested within a period of 30 days is about $15 \times 12 \times 6 \mathrm{~mm}^{3}$. 


\subsection{Single Crystal and Powder XRD Studies}

Single crystal XRD studies for grown DAHP crystal were carried out using single crystal $\mathrm{X}$-ray diffractometer with graphite monochormated $\mathrm{MoK}_{\alpha}$ radiation and the structure was solved by single crystal XRD analysis by direct method and refined by the full matrix-leastsquare technique using SHELXL program. The obtained data from single crystal XRD studies are presented in the table 1. From the data, it is observed that the grown crystals crystallize in monoclinic system with the space group $\mathrm{P} 2_{1} / \mathrm{c}$. The number of molecules per unit cell (Z) for DAHP crystal is found to be 4 . The unit cell parameters of DAHP crystals obtained in this work are very close agreement with reported work[8]. Powder XRD pattern of DAHP crystal is shown in Fig. 3. The well-defined peaks at specific $2 \Theta$ values show high crystallinity of the grown crystals. All the reflections of powder XRD pattern of the sample were indexed using the TREOR software package following the procedure of Lipson and Steeple [18]. The (hkl) planes satisfy the general reflection conditions of space group observed from the structure determination of the crystal. The obtained lattice parameters from powder XRD studies using UNITCELL software package are observed to be in close agreement with data obtained from single crystal XRD studies.

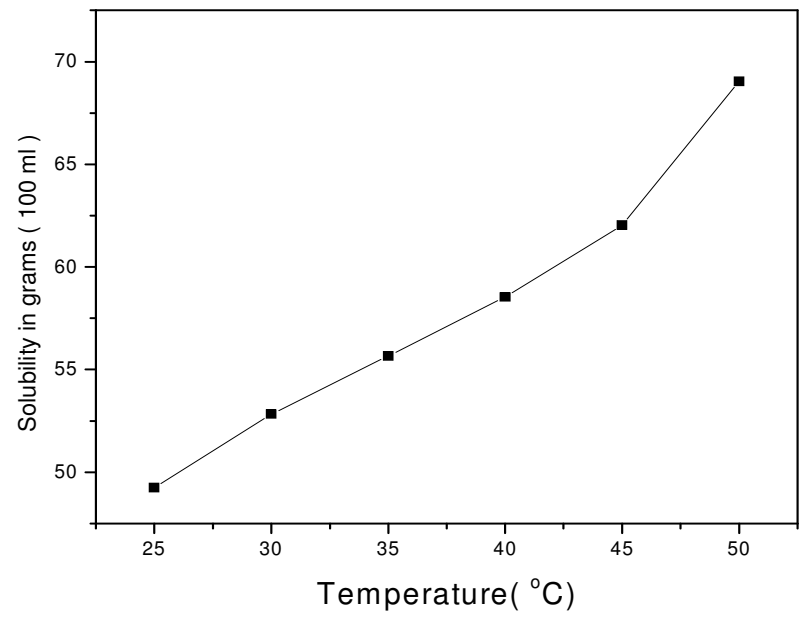

Fig.1: Variation of solubility with temperature for DAHP

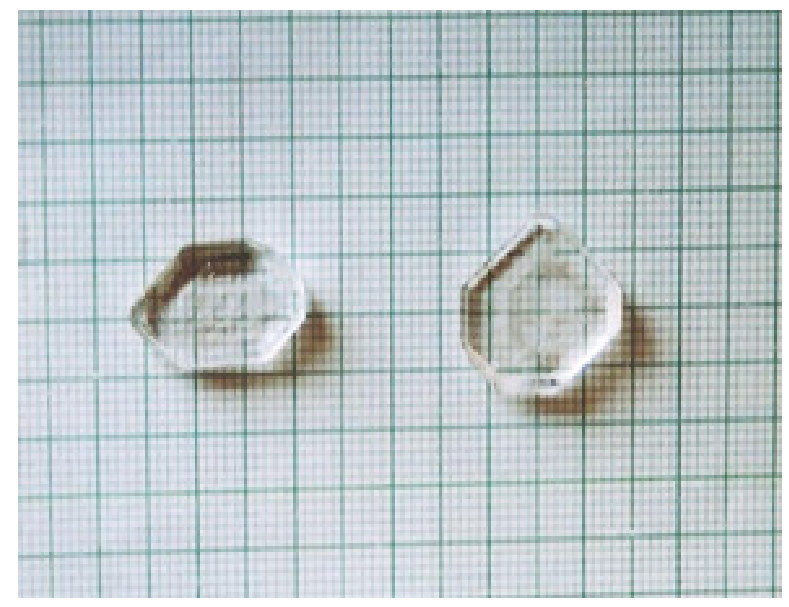

Fig.2: Harvested DAHP crystals 


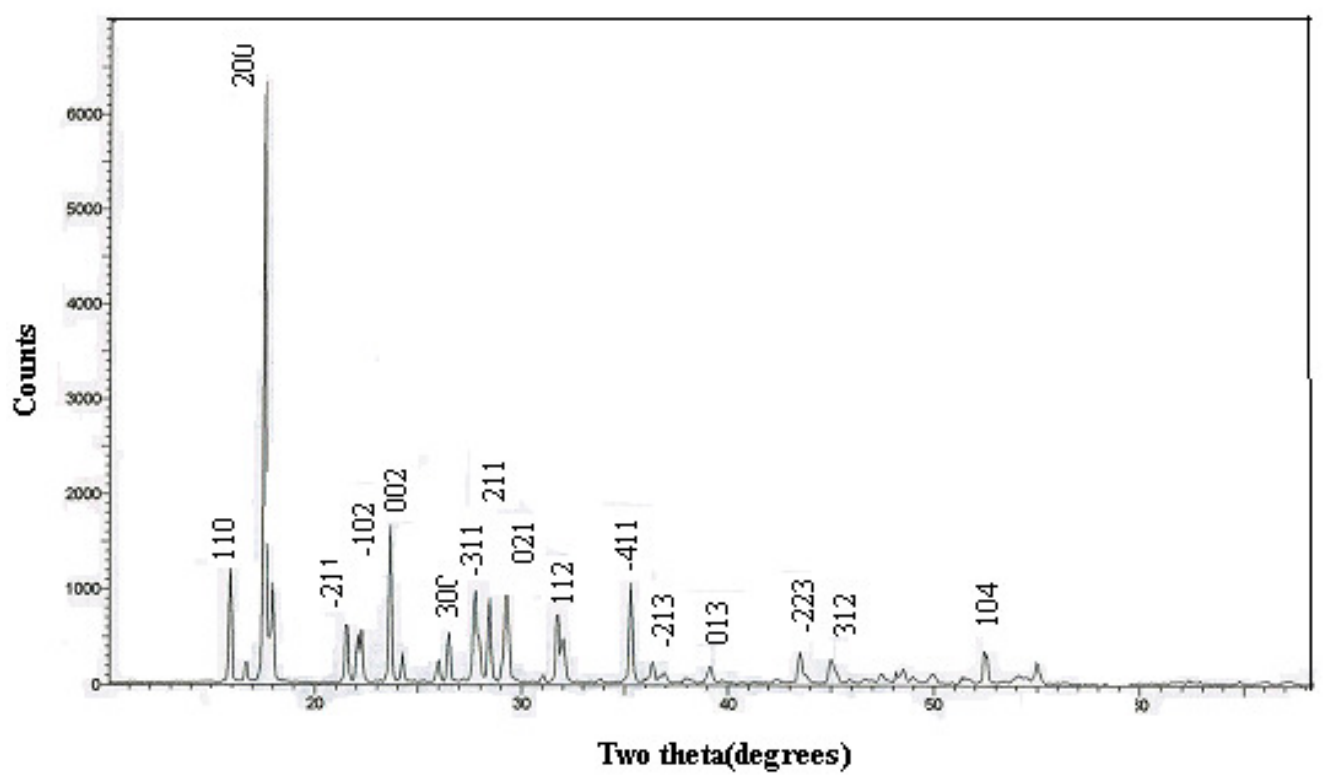

Fig.3: Powder XRD pattern of DAHP crystals

\subsection{Measurement of Density and Melting Point}

The value of density of DAHP crystal was measured by floatation method and it was found to be $1.6183 \mathrm{~g} / \mathrm{cc}$. This value is well matched with the reported value [19]. The density was also calculated from the crystallographic XRD data using the relation $\rho=(\mathrm{MZ}) /(\mathrm{NV})$ where $\mathrm{M}$ is the molecular weight of DAHP crystal, $\mathrm{Z}$ is the number of molecules per unit cell, $\mathrm{N}$ is Avogadro's number and V is volume of the unit cell and it was found to be $1.620 \mathrm{~g} / \mathrm{cc}$. Using the melting point apparatus, the value of melting point of DAHP crystal was found to be $202{ }^{\circ} \mathrm{C}$. The values of density is in good agreement with the data obtained from XRD studies.

Table 1 : Single crystal XRD data for DAHP crystal

\begin{tabular}{|c|c|}
\hline Chemical formula & ( NH4)2 H PO4 \\
\hline Molecular weight & 132.05 \\
\hline Crystal system & Monoclinic \\
\hline Space group & $\mathrm{P} 21 / \mathrm{c}$ \\
\hline $\mathrm{a}(\AA)$ & $10.923(4)$ \\
\hline $\mathrm{b}(\AA)$ & $6.645(2)$ \\
\hline 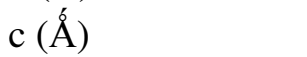 & $8.041(2)$ \\
\hline$\alpha$ & $90^{\circ}$ \\
\hline$\beta$ & $112.06^{\circ}$ \\
\hline$\gamma$ & $90^{\circ}$ \\
\hline V $(\AA ̊)) 3$ & $540.91(3)$ \\
\hline $\mathrm{Z}$ & 4 \\
\hline Density & $1.620 \mathrm{~g} / \mathrm{cc}$ \\
\hline
\end{tabular}




\subsection{UV-Visible Transmittance Studies}

Usually an NLO material must have a wide transparency window and this could be checked by recording an absorption or transmittance spectrum of the sample in UV-visibleIR region. The optical transmittance spectrum of DAHP crystal in the wavelength range $190-1100 \mathrm{~nm}$ is recorded and it is shown in Fig. 4. The study of the absorption edge is essential in connection with the theory of electronic structure, which leads to the prediction of whether the band structure is affected near the band extreme. From the transmittance spectra, it is noticed that DAHP crystal has high transmittance in the entire visible-NIR region of the spectrum. The high transmission in the entire visible region and short cut off wavelength facilitates the grown crystal to be a potential nonlinear optical material for second harmonic and third harmonic of Nd:YAG laser [20]. A sharp fall in the transmittance is observed at $196 \mathrm{~nm}$ for the grown DAHP crystal and this corresponds to the fundamental absorption. Absorption in the near ultraviolet region arises from electronic transitions associated within the sample. Using the formula $E_{g}=1240 / \lambda(\mathrm{nm})$, the band gap of the sample is calculated to be $6.326 \mathrm{eV}$.

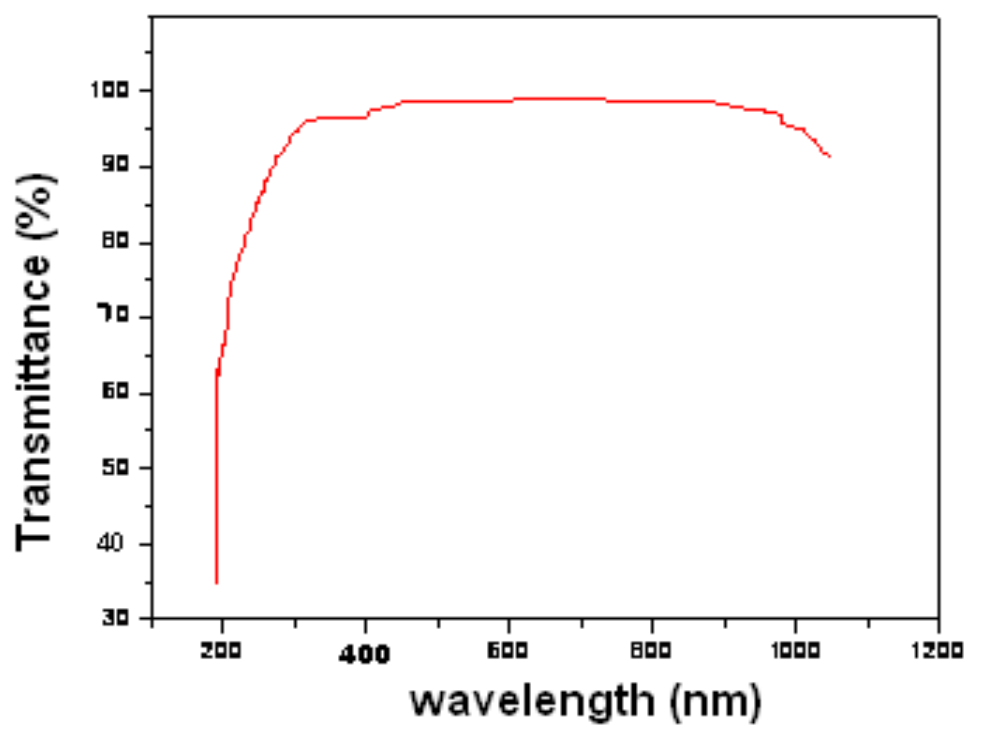

Fig.4: UV-Visible-NIR transmittance spectrum for DAHP crystal

\subsection{Microhardness Studies}

Microhardness studies for the grown DAHP crystal were performed at room temperature (30 ${ }^{\circ} \mathrm{C}$ ) to determine microhardness number and hence the mechanical strength and this study plays an important role in the fabrication of opto-electronic devices. The hardness of a material is a measure of its resistance to plastic deformation. In an ideal crystal, the hardness value should be independent of applied load. But in a real crystal, the load dependence is observed. This is due to normal indentation size effect (ISE) [21]. The variation of microhardness number $\left(\mathrm{H}_{\mathrm{V}}\right)$ with load applied to the plane (100) of the sample is displayed in Fig. 5 and it is noticed that Vickers hardness number $\left(\mathrm{H}_{\mathrm{v}}\right)$ increases with 
the applied load satisfying the indentation size effect. The maximum value of microhardness of DAHP crystal is observed to be $68.55 \mathrm{~kg} / \mathrm{mm}^{3}$ at $160 \mathrm{~g}$, beyond this load significant cracking occurs which may be due to the release of internal stresses produced within the indentation [22].

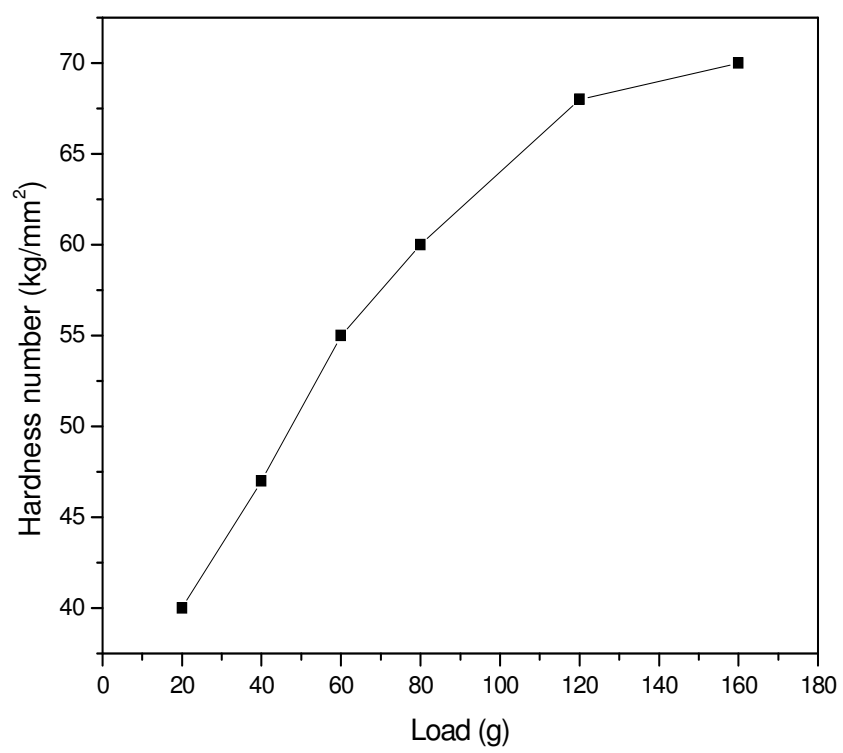

Fig.5: Variation of microhardness number with load for DAHP crystal

\subsection{Dielectric Characterization}

The dielectric parameters like dielectric constant $\left(\varepsilon_{\mathrm{r}}\right)$ and dielectric loss $((\tan \delta))$ are the basic electrical properties of solids. The measurement of dielectric constant and loss as a function of frequency and temperature gives the ideas of electrical processes that are taking place in material and these parameters were measured on (100) face of the sample. Variations of dielectric constant and dielectric loss of DAHP crystal at frequencies $100 \mathrm{~Hz}$ and $1 \mathrm{KHz}$ and in the temperature range $30-150{ }^{\circ} \mathrm{C}$ are displayed in Figs. 6 and 7. The results suggest that the dielectric constant and loss strongly depend on the frequency of a.c. signal and the temperature of the sample. It is observed from the figures that both dielectric constant and loss are found to be more at $100 \mathrm{~Hz}$ than those at $1 \mathrm{KHz}$ and observed to be increased when temperature of DAHP crystal is increased. The high values of dielectric constant and loss at $100 \mathrm{~Hz}$ are ascribed to space charge polarization. The dielectric constant of a material is known to consist of contributions from electronic, ionic, dipolar and space change polarizations, each dominating in a particular frequency range. It is well established that the space charge polarization is very predominant at lower frequencies. This polarization is known to arise from charged defects present and also due to the creation and distribution of dipoles either within the bulk or at the surface of the crystal. The dipolar orientational effect can sometimes be seen in some materials up to $10^{10} \mathrm{~Hz}$. The ionic and electronic polarizations always exist below $10{ }^{13} \mathrm{~Hz}$. The nature of decrease of $\varepsilon_{\mathbf{r}}$ and $(\tan \delta$ ) with frequency suggests that the grown DAHP crystal seem to contain dipoles of continuously 
varying relaxation times. Since the dipoles of larger relaxation times are not able to respond to $1 \mathrm{KHz}$, the dielectric constant $\left(\varepsilon_{\mathbf{r}}\right)$ and loss tangent $((\tan \delta))$ are low at $1 \mathrm{KHz}$ compared to $100 \mathrm{~Hz}$. Variation of the dielectric parameters with temperature is generally attributed to the crystal expansion, the electronic, space charge and ionic polarizations and the presence of impurities and crystal defects. The increase in the values of dielectric parameters at higher temperatures is mainly attributed to the thermally generated charge carriers and charged dipoles. As far as polarization is concerned, the increase in dielectric constant with temperature is essentially due to the temperature variation of ionic and space charge polarizations and not due to the temperature variation of orientational polarization [23-24].

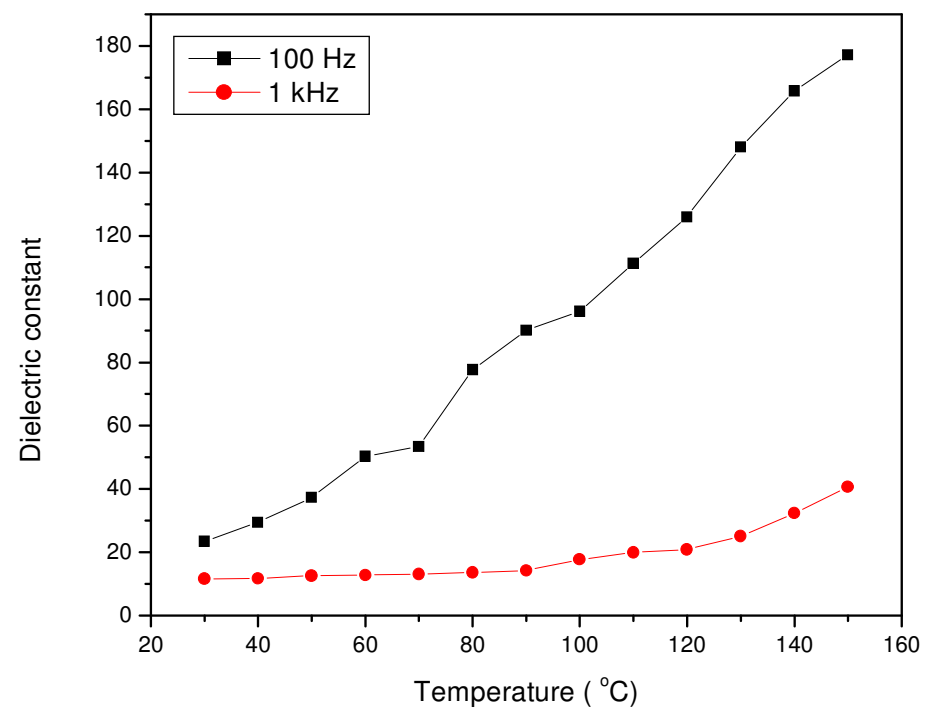

Fig.6: Variation of dielectric constant with temperature at frequencies of $100 \mathrm{~Hz}$ and $1 \mathrm{KHz}$ for DAHP crystal

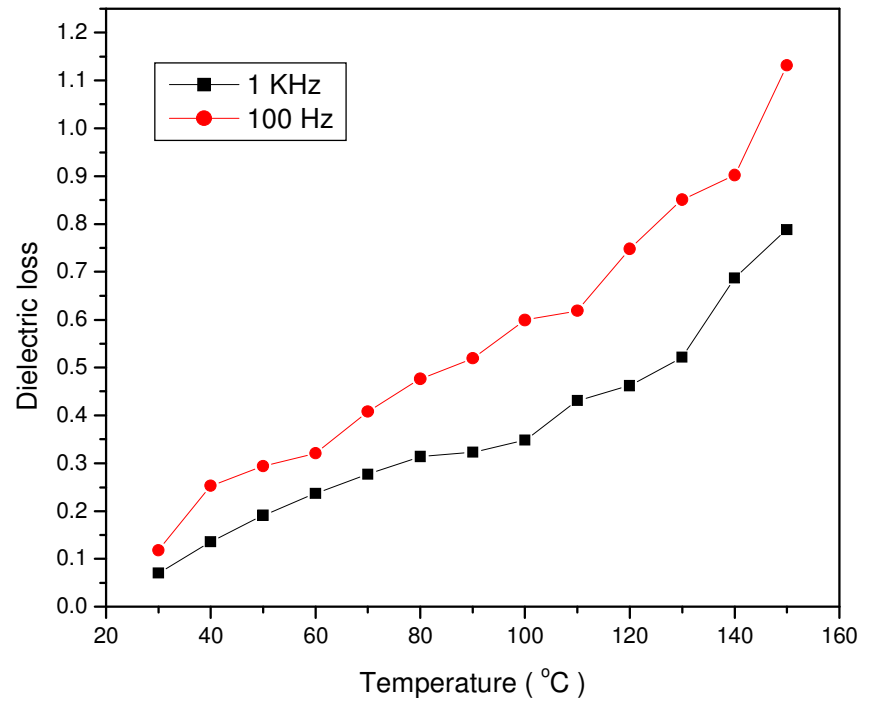

Fig.7: Variation of dielectric loss with temperature at frequencies of $100 \mathrm{~Hz}$ and $1 \mathrm{KHz}$ for DAHP crystal 


\subsection{Second Harmonic Generation (SHG) Test}

To confirm the nonlinear optical property of sample, powder form of DAHP crystals was subjected to NLO test obeying the Kurtz and Perry powder method [13] and Second Harmonic Generation (SHG) was confirmed by emission of green light $(\lambda=532 \mathrm{~nm})$. From the study, it was observed that the SHG relative efficiency of DAHP sample was found to be about 0.9 times as that of KDP. The good optical transmittance in the visible-NIR region and SHG conversion efficiency makes the grown crystal a potential material for device applications in optoelectronics.

\section{CONCLUSIONS}

Solubility of Diammonium Hydrogen Phosphate (DAHP) in de-ionized water was determined at various temperatures and optical quality single crystals of DAHP were grown by solution growth method with slow solvent evaporation technique. The grown crystals are transparent and with well defined external appearance. Single crystal and powder X-ray diffraction (XRD) methods reveal that the structure of the grown DAHP crystal belongs to monoclinic. The values of density and melting point of DAHP crystal are measured to be $1.618 \mathrm{~g} / \mathrm{cc}$ and $202{ }^{\circ} \mathrm{C}$ respectively. It is observed that the measured value of density of DAHP crystal by floatation method is well coincided with the density calculated from XRD data. UV-visible-NIR transmittance spectral studies for DAHP crystal show that the material has good optical transmittance in the entire visible region and low cut-off wavelength and hence it may be suitable for device fabrication in optoelectronics. Dielectric constant and dielectric loss tangent $(\tan \delta)$ measurements were carried out for the grown DAHP crystal at frequencies of $100 \mathrm{~Hz}$ and $1 \mathrm{KHz}$ and in the temperature range 30

- $150{ }^{\circ} \mathrm{C}$ and these values show the normal behaviour of insulating-type materials like DAHP. Vickers microhardness study shows that the DAHP crystal has high hardness values, revealing the reasonable mechanical strength of the material. The relative SHG efficiency of the grown sample was found to be about 0.9 times as that of KDP.

\section{ACKNOWLEDGEMENTS}

The authors are thankful to the staff members of IIT, Chennai, M.K.University, Madurai, Regional Research Laboratory, Trivananthapuram, Crescent Engineering College, Chennai for helping us to carry out various studies of the grown crystals of DAHP. Also the authors like to thank the management authorities of Aditanar College of Arts and Science, Tiruchendur and S.T. Hidnu College, Nagercoil for the constant encouragement to carry out the research in crystal growth.

\section{REFERENCE}

[1] S.B. Monaco, L.E. Davis, S.P. Velsko, F.T. Wang, D. Eimerl, A.J. Zalkin J. Crystal Growth 85 (1987) 252. 
[2] K. Meera, R. Muralidharan, R. Dhanasekaran, Prapun Manyum, P. Ramasamy J. Crystal Growth 263 (2004) 510.

[3] C. Justin Raj, S. Dinakaran, S. Krishnan, B. Milton Boaz, R. Robert, S. Jerome Das Optics Commun. 281 (2008) 2285.

[4] K. Ambujam , K. Rajarajan, S. Selvakumar, J. Madhavan, Gulam Mohamed, P. Sagayaraj Optical Mater. 29 (2007) 657.

[5] M.Gunasekaran, N.Vijayan, R.Ramesh Babu, R.Gopalakrishnan, P.Ramasamy J. Crystal Growth 244(2002) 194.

[6] N.Joseph John, P.Selvarajan, S. Sylvia, C.K.Mahadevan Mat.Manufact.Process. 22 (2007) 379.

[7] R.Navil Kumar and C.P.G. Vallaban J.Phys.Condens. Matter 1(1989) 6095.

[8] J.P.Smith, J.R.Lehr, W.E.Brown Acta Crystallogr. 10 (1957) 50.

[9] A.A.Khan, J.P.Roux, W.J.James Acta Crystallogr. B28(1978) 2065.

[10] A.Watton, E.C.Reynhardt, H.S. Sandhu, H.E.Petch J.Chem. Phys. 67(1977)887.

[11] G.Goobes , Vinodhkumar Raghunathanm Elizabeth A.Louie, James M.Gibson, Gregory L. Olsen, Gary P.Drobny Sold State Nucl. Mag. Resonance 29(2006) 242.

[12] P. Selvarajan, A. Siva dhas, T.H. Freeda, C.K. Mahadevan, Physica B 403(2008)4205.

[13] S.K. Kurtz , T.T. Perry J.Appl. Phys.39 (1968) 3798.

[14] C. Krishnan, P. Selvarajan, T.H. Freeda J. Crystal Growth 311 (2008)141.

[15] K.Kishan Rao, V.Surender and B.Saritha Rani Bull.Mater.Sci. 25(2002)641.

[16] C. Krishnan , P. Selvarajan, T.H. Freeda, C.K. Mahadevan Physica B 404 (2009) 289.

[17] S. Krishnan, C. Justin Raj, S. M. Navis Priya, R. Robert, S. Dinakaran, and S. Jerome Das Cryst. Res. Technol. 43, No. 8 (2008) 845.

[18] H. Lipson, H. Steeple, Interpretation of X-ray powder Diffraction Patterns, Fifth Ed, Macmillan, NewYork (1970), p.165.

[19] J.A.Dean, Lange's Hand book of Chemistry, $12^{\text {th }}$ edn., McGraw Hill , New York (1979), p.4-16.

[20] T. Balakrishnan, K. Ramamurthi Spectrochimica Acta Part A 72 (2009) 269.

[21] J.B. Charles, F.D. Gnanam J. Mater. Sci. Lett. 12 (1993) 1395.

[22] N. Theresita Shanthi, P. Selvarajan, C.K. Mahadevan, Curr. Appl. Phys. 9(2009)1155.

[23] K. V. Rao and A. Smakula J. Appl. Phys. 36(1965) 2031.

[24] P. Selvarajan, B.N. Das, H.B. Gon and K.V. Rao, J. Mater. Sci. 29 (1994) 4061. 